\title{
Nonlinear approximation of an ACQ-functional equation in nan-spaces
}

\author{
Hassan Azadi Kenary', Jung Rye Lee ${ }^{2^{*}}$ and Choonkil Park ${ }^{3}$
}

* Correspondence: jrlee@daejin.ac. $\mathrm{kr}$

${ }^{2}$ Department of Mathematics, Daejin University, Kyeonggi 487711, Korea

Full list of author information is available at the end of the article

\section{Abstract}

In this paper, using the fixed point and direct methods, we prove the generalized Hyers-Ulam stability of an additive-cubic-quartic functional equation in NAN-spaces. Mathematics Subject Classification (2010) 39B52.47H10.26E30-46S10-47S10

Keywords: generalized Hyers-Ulam stability, non-Archimedean normed space, fixed point method

\section{Introduction and preliminaries}

A classical question in the theory of functional equations is the following: "When is it true that a function which approximately satisfies a functional equation must be close to an exact solution of the equation?" If the problem accepts a solution, we say that the equation is stable. The first stability problem concerning group homomorphisms was raised by Ulam [1] in 1940. In the next year, Hyers [2] gave a positive answer to the above question for additive groups under the assumption that the groups are Banach spaces. In 1978, Rassias [3] proved a generalization of the Hyers' theorem for additive mappings. The result of Rassias has provided a lot of influence during the last three decades in the development of a generalization of the Hyers-Ulam stability concept. This new concept is known as generalized Hyers-Ulam stability or Hyers-UlamRassias stability of functional equations (see [4-8]). Furthermore, in 1994, a generalization of the Rassias' theorem was obtained by Găvruta [9] by replacing the bound $\varepsilon(\|$ $x||^{p}+\|y\|^{p}$ ) by a general control function $\phi(x, y)$.

The functional equation

$$
f(x+y)+f(x-y)=2 f(x)+2 f(y)
$$

is called a quadratic functional equation. In particular, every solution of the quadratic functional equation is said to be a quadratic mapping. In 1983, a generalized Hyers-Ulam stability problem for the quadratic functional equation was proved by Skof [10] for mappings $f: X \rightarrow Y$, where $X$ is a normed space and $Y$ is a Banach space. In 1984, Cholewa [11] noticed that the theorem of Skof is still true if the relevant domain $X$ is replaced by an Abelian group and, in 2002, Czerwik [12] proved the generalized Hyers-Ulam stability of the quadratic functional equation. 
The stability problems of several functional equations have been extensively investigated by a number of authors, and there are many interesting results concerning this problem (see [13-32]).

In 1897, Hensel [33] has introduced a normed space that does not have the Archimedean property. It turned out that non-Archimedean spaces have many nice applications (see [34-37]).

Now, we give some definitions and lemmas for the main results in this paper.

A valuation is a function $|\cdot|$ from a field $\mathbb{K}$ into $[0, \infty)$ such that, for all $r, s \in \mathbb{K}$, the following conditions hold:

(a) $|r|=0$ if and only if $r=0$;

(b) $|r s|=|r||s|$;

(c) $|r+s| \leq|r|+|s|$.

A field $\mathbb{K}$ is called a valued field if $\mathbb{K}$ carries a valuation. The usual absolute values of $\mathbb{R}$ and $\mathbb{C}$ are examples of valuations.

Let us consider a valuation that satisfies a stronger condition than the triangle inequality. If the triangle inequality is replaced by

$$
|r+s| \leq \max \{|r|,|s|\}
$$

for all $r, s \in \mathbb{K}$, then the function $|\cdot|$ is called a non-Archimedean valuation and the field is called a non-Archimedean field. Clearly, $|1|=|-1|=1$ and $|n| \leq 1$ for all $n \in$ $\mathbb{N}$. A trivial example of a non-Archimedean valuation is the function $|\cdot|$ taking everything except for 0 into 1 and $|0|=0$.

Definition 1.1. Let $X$ be a vector space over a field $\mathbb{K}$ with a non-Archimedean valuation $|\cdot|$. A function $\|\cdot\|: X \rightarrow[0, \infty)$ is called a non-Archimedean norm if the following conditions hold:

(a) $\|x\|=0$ if and only if $x=0$ for all $x \in X$;

(b) $\|r x||=|r|\| x \|$ for all $r \in K$ and $x \in X$;

(c) the strong triangle inequality holds:

$$
\|x+y\| \leq \max \{\|x\|,\|y\|\}
$$

for all $x, y \in X$.

Then $(X,\|\cdot\|)$ is called a non-Archimedean normed space (briefly NAN-space).

Definition 1.2. Let $\left\{x_{n}\right\}$ be a sequence in a non-Archimedean normed space $X$.

(1) The sequence $\left\{x_{n}\right\}$ is called a Cauchy sequence if, for any $\varepsilon>0$, there is a positive integer $N$ such that

$$
|| x_{n}-x_{m} \| \leq \varepsilon
$$

for all $n, m \geq N$.

(2) The sequence $\left\{x_{n}\right\}$ is said to be convergent if, for any $\varepsilon>0$, there are a positive integer $N$ and $x \in X$ such that

$$
\left\|x_{n}-x\right\| \leq \varepsilon
$$

for all $n \geq N$. Then, the point $x \in X$ is called the limit of the sequence $\left\{x_{n}\right\}$, which is denoted by $\lim _{n \rightarrow \infty} x_{n}=x$.

(3) If every Cauchy sequence in $X$ converges, then the non-Archimedean normed space $X$ is called a non-Archimedean Banach space. 
Note that $\left\|x_{n}-x_{m}\right\| \leq \max \left\{|| x_{j+1}-x_{j} \|: m \leq j \leq n-1\right\}$ for all $m, n \geq 1$ with $n>m$.

Definition 1.3. Let $X$ be a set. A function $d: X \times X \rightarrow[0, \infty]$ is called a generalized metric on $X$ if $d$ satisfies the following conditions:

(a) $d(x, y)=0$ if and only if $x=y$ for all $x, y \in X$;

(b) $d(x, y)=d(y, x)$ for all $x, y \in X$;

(c) $d(x, z) \leq d(x, y)+d(y, z)$ for all $x, y, z \in X$.

Theorem 1.1. $[38,39]$ Let $(X, d)$ be a complete generalized metric space and $J: X \rightarrow X$ be a strictly contractive mapping with Lipschitz constant $L<1$. Then, for all $x \in X$, either

$$
d\left(J^{n} x, J^{n+1} x\right)=\infty
$$

for all nonnegative integers $n$ or there exists a positive integer $n_{0}$ such that

(a) $d\left(J^{n} x, J^{n+1} x\right)<\infty$ for all $n_{0} \geq n_{0}$;

(b) the sequence $\left\{J^{n} x\right\}$ converges to a fixed point $y^{*}$ of $J$;

(c) $y^{*}$ is the unique fixed point of $J$ in the set $Y=\left\{y \in X: d\left(J^{n_{0}} x, y\right)<\infty\right\}$;

(d) $d\left(y, y^{*}\right) \leq \frac{1}{1-L} d(y, J y)$ for all $y \in Y$.

In this paper, using the fixed point and direct methods, we prove the generalized Hyers-Ulam stability of the following functional equation

$$
\begin{aligned}
11 f(x+2 y)+11 f(x-2 y)= & 44\{f(x+y)+f(x-y)\}+12 f(3 y) \\
& -48 f(2 y)+60 f(y)-66 f(x)
\end{aligned}
$$

in non-Archimedean normed spaces.

\section{Non-Archimedean stability of the equation (1.1): a fixed point method- odd case}

Using the fixed point alternative approach, we prove the generalized Hyers-Ulam stability of functional Equation (1.1) in non-Archimedean normed spaces for an odd case.

In [40], Lee et al. considered the following quartic functional equation:

$$
f(2 x+y)+f(2 x-y)=4\{f(x+y)+f(x-y)\}+24 f(x)-6 f(y)
$$

It is easy to show that the function $f(x)=x^{4}$ satisfies the functional Equation (2.1), which is called a quartic functional equation and every solution of the quartic functional equation is said to be a quartic mapping.

One can easily show that an even mapping $f: X \rightarrow Y$ satisfies (1.1) if and only if the even mapping $f: X \rightarrow Y$ is a quartic mapping, that is,

$$
f(2 x+y)+f(2 x-y)=4\{f(x+y)+f(x-y)\}+24 f(x)-6 f(y)
$$

and an odd mapping $f: X \rightarrow Y$ satisfies (1.1) if and only if the odd mapping $f: X \rightarrow$ $Y$ is a additive-cubic mapping, that is,

$$
f(2 x+y)+f(2 x-y)=4\{f(x+y)+f(x-y)\}-6 f(x)
$$

It was shown in [[41], Lemma 2.2] that $g(x)=f(2 x)-2 f(x)$ and $h(x)=f(2 x)-8 f(x)$ are cubic and additive, respectively, and that $f(x):=\frac{1}{16} g(x)-\frac{1}{16} h(x)$. 
For a given mapping $f: X \rightarrow Y$, we define

$$
\begin{aligned}
\Phi_{f}(x, y)= & 11 f(x+2 y)+11 f(x-2 y)-44\{f(x+y)+f(x-y)\} \\
& -12 f(3 y)+48 f(2 y)-60 f(y)+66 f(x)
\end{aligned}
$$

for all $x, y \in X$.

Using the fixed point method, we prove the generalized Hyers-Ulam stability of the functional equation $\Phi_{f}(x, y)=0$ in non-Archimedean normed spaces: an odd case.

Throughout this section, let $|8| \neq 1$.

Theorem 2.1. Let $X$ be a non-Archimedean normed space and $Y$ a non-Archimedean Banach space. Assume that $\gamma: X^{2} \rightarrow[0, \infty)$ is a function such that there exists an $L<1$ with

$$
\gamma\left(\frac{x}{2}, \frac{y}{2}\right) \leq \frac{L}{|8|} \gamma(x, y)
$$

for all $x, y \in X$. If $f: X \rightarrow Y$ is an odd mapping satisfying

$$
\left\|\Phi_{f}(x, y)\right\| \leq \gamma(x, y)
$$

for all $x, y \in X$, then the limit

$$
C(x):=\lim _{n \rightarrow \infty} 8^{n}\left(f\left(\frac{x}{2^{n-1}}\right)-2 f\left(\frac{x}{2^{n}}\right)\right)
$$

exists for all $x \in X$ and defines a unique cubic mapping $C: X \rightarrow Y$ such that

$$
\|f(2 x)-2 f(x)-C(x)\| \leq \frac{L}{|8|-|8| L} \max \left\{\frac{1}{|11|} \gamma(2 x, x),\left|\frac{14}{33}\right| \gamma(x, 0)\right\} .
$$

Proof. Putting $x=0$ in (2.5), we have

$$
\|12 f(3 \gamma)-48 f(2 \gamma)+60 f(y)\| \leq \gamma(y, 0)
$$

for all $y \in X$.

Replacing $x$ by $2 y$ in (2.5), we get

$$
\|11 f(4 y)-56 f(3 y)+114 f(2 \gamma)-104 f(\gamma)\| \leq \gamma(2 y, \gamma)
$$

for all $y \in X$. By (2.7) and (2.8), we have

$$
\begin{aligned}
\|f(4 y)-10 f(2 \gamma)+16 f(\gamma)\|= & \| \frac{1}{11}[11 f(4 y)-56 f(3 \gamma)+114 f(2 \gamma)-104 f(\gamma)] \\
& +\frac{14}{33}[12 f(3 \gamma)-48 f(2 \gamma)+60 f(y)] \| \\
\leq & \max \left\{\frac{1}{|11|} \gamma(2 \gamma, \gamma),\left|\frac{14}{33}\right| \gamma(\gamma, 0)\right\}
\end{aligned}
$$

for all $y \in X$. Letting $y:=\frac{x}{2}$ and $g(x):=f(2 x)-2 f(x)$ for all $x \in X$, we get

$$
\left\|g(x)-8 g\left(\frac{x}{2}\right)\right\| \leq \max \left\{\frac{1}{|11|} \gamma\left(x, \frac{x}{2}\right),\left|\frac{14}{33}\right| \gamma\left(\frac{x}{2}, 0\right)\right\} .
$$

Consider the set

$$
S:=\{g: X \rightarrow Y\}
$$


and the generalized metric $d$ in $S$ defined by

$$
d(f, g)=\inf _{\mu \in(0,+\infty)}\left\{\|g(x)-h(x)\| \leq \mu \max \left\{\frac{1}{|11|} \gamma(2 x, x),\left|\frac{14}{33}\right| \gamma(x, 0)\right\}, \forall x \in X\right\},
$$

where $\inf \varnothing=+\infty$. It is easy to show that $(S, d)$ is complete (see [[42], Lemma 2.1]). Now, we consider a linear mapping $J: S \rightarrow S$ such that

$$
J g(x):=8 g\left(\frac{x}{2}\right)
$$

for all $x \in X$. Let $g, h \in S$ be such that $d(g, h)=\varepsilon$. Then we have

$$
\|g(x)-h(x)\| \leq \varepsilon \max \left\{\frac{1}{|11|} \gamma(2 x, x),\left|\frac{14}{33}\right| \gamma(x, 0)\right\}
$$

for all $x \in X$ and so

$$
\begin{aligned}
\|J g(x)-J h(x)\| & =\left\|8 g\left(\frac{x}{2}\right)-8 h\left(\frac{x}{2}\right)\right\| \\
& \leq|8| \max \left\{\frac{1}{|11|} \gamma\left(x, \frac{x}{2}\right),\left|\frac{14}{33}\right| \gamma\left(\frac{x}{2}, 0\right)\right\} \\
& \leq|8| \cdot \frac{L}{|8|} \varepsilon \max \left\{\frac{1}{|11|} \gamma(2 x, x),\left|\frac{14}{33}\right| \gamma(x, 0)\right\}
\end{aligned}
$$

for all $x \in X$. Thus $d(g, h)=\varepsilon$ implies that $d(J g, J h) \leq L \varepsilon$. This means that

$$
d(J g, J h) \leq L d(g, h)
$$

for all $g, h \in S$. It follows from (2.10) that

$$
d(g, J g) \leq \frac{L}{|8|} .
$$

By Theorem 1.1, there exists a mapping $C: X \rightarrow Y$ satisfying the following:

(1) $C$ is a fixed point of $J$, that is,

$$
\frac{1}{8} C(x)=C\left(\frac{x}{2}\right)
$$

for all $x \in X$. The mapping $C$ is a unique fixed point of $J$ in the set

$$
\Omega=\{h \in S: d(g, h)<\infty\} .
$$

This implies that $C$ is a unique mapping satisfying (2.13) such that there exists $\mu \in$ $(0, \infty)$ satisfying

$$
\|g(x)-C(x)\| \leq \mu \max \left\{\frac{1}{|11|} \gamma(2 x, x),\left|\frac{14}{33}\right| \gamma(x, 0)\right\}
$$

for all $x \in X$.

(2) $d\left(J^{n} g, C\right) \rightarrow 0$ as $n \rightarrow \infty$. This implies the equality

$$
\lim _{n \rightarrow \infty} 8^{n} g\left(\frac{x}{2^{n}}\right)=\lim _{n \rightarrow \infty} 8^{n}\left(f\left(\frac{x}{2^{n-1}}\right)-2 f\left(\frac{x}{2^{n}}\right)\right)=C(x)
$$

for all $x \in X$. 
(3) $d(g, C) \leq \frac{d(g, I g)}{1-L}$ with $g \in \Omega$, which implies the inequality

$$
d(g, C) \leq \frac{L}{|8|-|8| L} .
$$

This implies that the inequality (2.6) holds.

Since $\Phi_{g}(x, y)=\Phi_{f}(2 x, 2 y)-2 \Phi_{f}(x, y)$, using (2.4) and (2.5), we have

$$
\begin{aligned}
\left\|\Phi_{C}(x, y)\right\| & =\lim _{n \rightarrow \infty}|8|^{n}\left\|\Phi_{g}\left(\frac{x}{2^{n}}, \frac{y}{2^{n}}\right)\right\| \\
& =\lim _{n \rightarrow \infty}|8|^{n}\left\|\Phi_{f}\left(\frac{x}{2^{n-1}}, \frac{y}{2^{n-1}}\right)-2 \Phi_{f}\left(\frac{x}{2^{n}}, \frac{y}{2^{n}}\right)\right\| \\
& \leq \lim _{n \rightarrow \infty}|8|^{n} \max \left\{\left\|\Phi_{f}\left(\frac{x}{2^{n-1}}, \frac{y}{2^{n-1}}\right)\right\|,|2|\left\|\Phi_{f}\left(\frac{x}{2^{n}}, \frac{y}{2^{n}}\right)\right\|\right\} \\
& \leq \lim _{n \rightarrow \infty}|8|^{n} \max \left\{\gamma\left(\frac{x}{2^{n-1}}, \frac{y}{2^{n-1}}\right),|2| \gamma\left(\frac{x}{2^{n}}, \frac{y}{2^{n}}\right)\right\} \\
& \leq \lim _{n \rightarrow \infty}|8|^{n} \max \left\{\frac{L^{n-1}}{|8|^{n-1}} \gamma(x, y), \frac{|2| L^{n}}{|8|^{n}} \gamma(x, y)\right\} \\
& =0
\end{aligned}
$$

for all $x, y \in X$ and $n \geq 1$ and so $\left\|\Phi_{C}(x, y)\right\|=0$ for all $x, y \in X$. Therefore, the mapping $C: X \rightarrow Y$ is cubic. This completes the proof.

Corollary 2.1. Let $\theta \geq 0$ and $r$ be a real number with $r>1$. Let $f: X \rightarrow Y$ be an odd mapping satisfying

$$
\left\|\Phi_{f}(x, y)\right\| \leq \theta\left(\|x\|^{r}+\|y\|^{r}\right)
$$

for all $x, y \in X$. Then the limit $C(x)=\lim _{n \rightarrow \infty} 8^{n}\left(f\left(\frac{x}{2^{n-1}}\right)-2 f\left(\frac{x}{2^{n}}\right)\right)$ exists for all $x \in$ $X$ and $C: X \rightarrow Y$ is a unique cubic mapping such that

$$
\|f(2 x)-2 f(x)-C(x)\| \leq \frac{|8|^{r}}{|8|-|8|^{r+1}} \max \left\{\frac{\left.\left(|2|^{r}+1\right) \theta|| x\right|^{r}}{|11|},\left.\left|\frac{14}{33}\right| \theta|| x\right|^{r}\right\}
$$

for all $x \in X$.

Proof. The proof follows from Theorem 2.1 if we take

$$
\gamma(x, y)=\theta\left(\|x\|^{r}+\|y\|^{r}\right)
$$

for all $x, y \in X$. In fact, if we choose $L=|8|^{r}$, then we get the desired result.

Theorem 2.2. Let $X$ be a non-Archimedean normed space and $Y$ a non-Archimedean Banach space. Assume that $\gamma: X^{2} \rightarrow[0, \infty)$ is a function such that there exists an $L<1$ with

$$
\gamma(2 x, 2 y) \leq|8| L \gamma(x, y)
$$

for all $x, y \in X$. If $f: X \rightarrow Y$ is an odd mapping satisfying (2.5), then the limit

$$
C(x)=\lim _{n \rightarrow \infty} \frac{f\left(2^{n+1} x\right)-2 f\left(2^{n} x\right)}{8^{n}}
$$

exists for all $x \in X$ and defines a unique cubic mapping $C: X \rightarrow Y$ such that

$$
\|f(2 x)-2 f(x)-C(x)\| \leq \frac{1}{|8|-|8| L} \max \left\{\frac{1}{|11|} \gamma(2 x, x),\left|\frac{14}{33}\right| \gamma(x, 0)\right\} .
$$


Proof. Let $(S, d)$ be the generalized metric space defined in the proof of Theorem 2.1. Consider the mapping $J:(S, d) \rightarrow(S, d)$ such that

$$
J g(x):=\frac{1}{8} g(2 x)
$$

for all $x \in X$.

Proceeding as in the proof of Theorem 2.1, we find that $d(g, h)=\varepsilon$ implies that $d(J g$, $J h) \leq L \varepsilon$. This means that $d(J g, J h) \leq L d(g, h)$ for all $g, h \in S$.

It follows from (2.10) that

$$
\left\|\frac{g(2 x)}{8}-g(x)\right\| \leq \frac{1}{|8|} \max \left\{\frac{1}{|11|} \gamma(2 x, x),\left|\frac{14}{33}\right| \gamma(x, 0)\right\}
$$

for all $x \in X$. So

$$
d(g, J g) \leq \frac{1}{|8|}
$$

By Theorem 1.1, there exists a mapping $C: X \rightarrow Y$ satisfying the following:

(1) $C$ is a fixed point of $J$, that is,

$$
8 C(x)=C(2 x)
$$

for all $x \in X$. The mapping $C$ is a unique fixed point of $J$ in the set

$$
\Omega=\{h \in S: d(g, h)<\infty\} .
$$

This implies that $C$ is a unique mapping satisfying (2.20) such that there exists $\mu \in$ $(0, \infty)$ satisfying

$$
\|g(x)-C(x)\| \leq \mu \max \left\{\frac{1}{|11|} \gamma(2 x, x),\left|\frac{14}{33}\right| \gamma(x, 0)\right\}
$$

for all $x \in X$.

(2) $d\left(J^{n} g, C\right) \rightarrow 0$ as $n \rightarrow \infty$. This implies the equality

$$
\lim _{n \rightarrow \infty} \frac{g\left(2^{n} x\right)}{8^{n}}=\lim _{n \rightarrow \infty} \frac{f\left(2^{n+1} x\right)-2 f\left(2^{n} x\right)}{8^{n}}=C(x)
$$

for all $x \in X$.

(3) $d(g, C) \leq \frac{d(g, g)}{1-L}$ with $g \in \Omega$, which implies the inequality

$$
d(g, C) \leq \frac{1}{|8|-|8| L} .
$$

This implies that the inequality (2.17) holds. The rest of the proof is similar to the proof of Theorem 2.1.

Corollary 2.2. Let $\theta \geq 0$ and $r$ be a real number with $0<r<1$. Let $f: X \rightarrow Y$ be an odd mapping satisfying (2.15). Then the limit $C(x)=\lim _{n \rightarrow \infty} \frac{f\left(2^{n+1} x\right)-2 f\left(2^{n} x\right)}{8^{n}}$ exists for all $x \in X$ and $C: X \rightarrow Y$ is a unique cubic mapping such that

$$
\|f(2 x)-2 f(x)-C(x)\| \leq \frac{|8|^{r}}{|8|^{r+1}-|8|^{2}} \max \left\{\frac{\left(|2|^{r}+1\right) \theta|| x||^{r}}{|11|},\left.\left|\frac{14}{33}\right| \theta|| x\right|^{r}\right\}
$$

for all $x \in X$. 
Proof. The proof follows from Theorem 2.2 if we take

$$
\gamma(x, y)=\theta\left(\|x\|^{r}+\|y\|^{r}\right)
$$

for all $x, y \in X$. In fact, if we choose $L=|8|^{1-r}$, then we get the desired result.

Theorem 2.3. Let $X$ be a non-Archimedean normed space and $Y$ a non-Archimedean Banach space. Assume that $\gamma: X^{2} \rightarrow[0, \infty)$ is a function such that there exists an $L<1$ with

$$
\gamma\left(\frac{x}{2}, \frac{y}{2}\right) \leq \frac{L}{|2|} \gamma(x, y)
$$

for all $x, y \in X$. If $f: X \rightarrow Y$ is an odd mapping satisfying (2.5), then the limit

$$
A(x):=\lim _{n \rightarrow \infty} 2^{n}\left(f\left(\frac{x}{2^{n-1}}\right)-8 f\left(\frac{x}{2^{n}}\right)\right)
$$

exists for all $x \in X$ and defines a unique additive mapping $A: X \rightarrow Y$ such that

$$
\| f(2 x)-8 f(x)-A(x)|| \leq \frac{L}{|2|-|2| L} \max \left\{\frac{1}{|11|} \gamma(2 x, x),\left|\frac{14}{33}\right| \gamma(x, 0)\right\} .
$$

Proof. Let $(S, d)$ be the generalized metric space defined in the proof of Theorem 2.1. Letting $y:=\frac{x}{2}$ and $h(x):=f(2 x)-8 f(x)$ for all $x \in X$ in (2.9), we get

$$
\left\|h(x)-2 h\left(\frac{x}{2}\right)\right\| \leq \max \left\{\frac{1}{|11|} \gamma\left(x, \frac{x}{2}\right),\left|\frac{14}{33}\right| \gamma\left(\frac{x}{2}, 0\right)\right\} .
$$

Now, we consider a linear mapping $J: S \rightarrow S$ such that

$$
J h(x):=2 h\left(\frac{x}{2}\right)
$$

for all $x \in X$. Let $g, h \in S$ be such that $d(g, h)=\varepsilon$. Then we have

$$
\|g(x)-h(x)\| \leq \varepsilon \max \left\{\frac{1}{|11|} \gamma(2 x, x),\left|\frac{14}{33}\right| \gamma(x, 0)\right\}
$$

for all $x \in X$ and so

$$
\begin{aligned}
\|J g(x)-J h(x)\|=\left\|2 g\left(\frac{x}{2}\right)-2 h\left(\frac{x}{2}\right)\right\| & \leq|2| \max \left\{\frac{1}{|11|} \gamma\left(x, \frac{x}{2}\right),\left|\frac{14}{33}\right| \gamma\left(\frac{x}{2}, 0\right)\right\} \\
& \leq|2| \cdot \frac{L}{|2|} \varepsilon \max \left\{\frac{1}{|11|} \gamma(2 x, x),\left|\frac{14}{33}\right| \gamma(x, 0)\right\}
\end{aligned}
$$

for all $x \in X$. Thus, $d(g, h)=\varepsilon$ implies that $d(J g, J h) \leq L \varepsilon$. This means that

$$
d(J g, J h) \leq L d(g, h)
$$

for all $g, h \in S$. It follows from (2.24) that

$$
d(g, J g) \leq \frac{L}{|2|}
$$

By Theorem 1.1, there exists a mapping $A: X \rightarrow Y$ satisfying the following: 
(1) $A$ is a fixed point of $J$, that is,

$$
\frac{1}{2} A(x)=A\left(\frac{x}{2}\right)
$$

for all $x \in X$. The mapping $A$ is a unique fixed point of $J$ in the set

$$
\Omega=\{h \in S: d(g, h)<\infty\} .
$$

This implies that $A$ is a unique mapping satisfying (2.27) such that there exists $\mu \in$ $(0, \infty)$ satisfying

$$
\|h(x)-A(x)\| \leq \mu \max \left\{\frac{1}{|11|} \gamma(2 x, x),\left|\frac{14}{33}\right| \gamma(x, 0)\right\}
$$

for all $x \in X$.

(2) $d\left(J^{n} h, A\right) \rightarrow 0$ as $n \rightarrow \infty$. This implies the equality

$$
\lim _{n \rightarrow \infty} 2^{n} h\left(\frac{x}{2^{n}}\right)=\lim _{n \rightarrow \infty} 2^{n}\left(f\left(\frac{x}{2^{n-1}}\right)-8 f\left(\frac{x}{2^{n}}\right)\right)=A(x)
$$

for all $x \in X$.

(3) $d(h, A) \leq \frac{d(h, J h)}{1-L}$ with $h \in \Omega$, which implies the inequality

$$
d(h, A) \leq \frac{L}{|2|-|2| L} .
$$

This implies that the inequality (2.23) holds. The rest of the proof is similar to the proof of Theorem 2.1.

Corollary 2.3. Let $\theta \geq 0$ and $r$ be a real number with $r>1$. Let $f: X \rightarrow Y$ be an odd mapping satisfying (2.15). Then, the limit $A(x)=\lim _{n \rightarrow \infty} 2^{n}\left(f\left(\frac{x}{2^{n-1}}\right)-8 f\left(\frac{x}{2^{n}}\right)\right)$ exists for all $x \in X$ and $A: X \rightarrow Y$ is a unique additive mapping such that

$$
\|f(2 x)-8 f(x)-A(x)\| \leq \frac{|2|^{r}}{|2|-|2|^{r+1}} \max \left\{\frac{\left(|2|^{r}+1\right) \theta\|x\|^{r}}{|11|},\left|\frac{14}{33}\right| \theta\|x\|^{r}\right\}
$$

for all $x \in X$.

Proof. The proof follows from Theorem 2.3 if we take

$$
\gamma(x, y)=\theta\left(\|x\|^{r}+\|y\|^{r}\right)
$$

for all $x, y \in X$. In fact, if we choose $L=|2|^{r}$, then we get the desired result.

Theorem 2.4. Let $X$ be a non-Archimedean normed space and $Y$ a non-Archimedean Banach space. Assume that $\gamma: X^{2} \rightarrow[0, \infty)$ is a function such that there exists an $L<1$ with

$$
\gamma(2 x, 2 y) \leq|2| L \gamma(x, y)
$$

for all $x, y \in X$. If $f: X \rightarrow Y$ is an odd mapping satisfying (2.5), then the limit

$$
A(x)=\lim _{n \rightarrow \infty} \frac{f\left(2^{n+1} x\right)-8 f\left(2^{n} x\right)}{2^{n}}
$$


exists for all $x \in X$ and defines a unique additive mapping $A: X \rightarrow Y$ such that

$$
\|f(2 x)-8 f(x)-A(x)\| \leq \frac{1}{|2|-|2| L} \max \left\{\frac{1}{|11|} \gamma(2 x, x),\left|\frac{14}{33}\right| \gamma(x, 0)\right\} .
$$

Proof. Let $(S, d)$ be the generalized metric space defined in the proof of Theorem 2.1. Consider the mapping $J:(S, d) \rightarrow(S, d)$ such that

$$
J g(x):=\frac{1}{2} g(2 x)
$$

for all $x \in X$. By (2.24), we obtain

$$
\left\|\frac{h(2 x)}{2}-g(x)\right\| \leq \frac{1}{|2|} \max \left\{\frac{1}{|11|} \gamma(2 x, x),\left|\frac{14}{33}\right| \gamma(x, 0)\right\}
$$

for all $x \in X$. So

$$
d(g, J g) \leq \frac{1}{|2|} .
$$

By Theorem 1.1, there exists a mapping $A: X \rightarrow Y$ satisfying the following:

(1) $A$ is a fixed point of $J$, that is,

$$
2 A(x)=A(2 x)
$$

for all $x \in X$. The mapping $A$ is a unique fixed point of $J$ in the set

$$
\Omega=\{h \in S: d(g, h)<\infty\} .
$$

This implies that $A$ is a unique mapping satisfying (2.33) such that there exists $\mu \in$ $(0, \infty)$ satisfying

$$
\|h(x)-A(x)\| \leq \mu \max \left\{\frac{1}{|11|} \gamma(2 x, x),\left|\frac{14}{33}\right| \gamma(x, 0)\right\}
$$

for all $x \in X$.

(2) $d\left(J^{n} h, A\right) \rightarrow 0$ as $n \rightarrow \infty$. This implies the equality

$$
\lim _{n \rightarrow \infty} \frac{h\left(2^{n} x\right)}{2^{n}}=\lim _{n \rightarrow \infty} \frac{f\left(2^{n+1} x\right)-8 f\left(2^{n} x\right)}{2^{n}}=A(x)
$$

for all $x \in X$.

(3) $d(h, A) \leq \frac{d(h, h)}{1-L}$ with $h \in \Omega$, which implies the inequality

$$
d(h, A) \leq \frac{1}{|2|-|2| L} .
$$

This implies that the inequality (2.30) holds. The rest of the proof is similar to the proof of Theorem 2.1. $\quad$

Corollary 2.4. Let $\theta \geq 0$ and $r$ be a real number with $0<r<1$. Let $f: X \rightarrow Y$ be an odd mapping satisfying (2.15). Then, the limit $A(x)=\lim _{n \rightarrow \infty} \frac{f\left(2^{n+1} x\right)-8 f\left(2^{n} x\right)}{2^{n}}$ exists for all $x \in X$ and $A: X \rightarrow Y$ is a unique additive mapping such that

$$
\|f(2 x)-8 f(x)-A(x)\| \leq \frac{1}{|2|-|2|^{r+2}} \max \left\{\frac{\left(|2|^{r}+1\right) \theta\|x\|^{r}}{|11|},\left|\frac{14}{33}\right| \theta\|x\|^{r}\right\}
$$


for all $x \in X$.

Proof. The proof follows from Theorem 2.4 if we take

$$
\gamma(x, y)=\theta\left(\|x\|^{r}+\|y\|^{r}\right)
$$

for all $x, y \in X$. In fact, if we choose $L=|2|^{r+1}$, then we get the desired result.

\section{Non-Archimedean stability of the equation (1.1): a fixed point method- even case}

Using the fixed point method, we prove the generalized Hyers-Ulam stability of the functional Equation (1.1) in non-Archimedean normed spaces for an even case. Throughout this section, let $|16| \neq 1$.

Theorem 3.1. Let $X$ be a non-Archimedean normed space and $Y$ a non-Archimedean Banach space. Assume that $\gamma: X^{2} \rightarrow[0, \infty)$ is a function such that there exists an $L<1$ with

$$
\gamma(2 x, 2 y) \leq|16| L \gamma(x, y)
$$

for all $x, y \in X$. If $f: X \rightarrow Y$ is an even mapping with $f(0)=0$ satisfying (2.5), then the limit

$$
Q(x):=\lim _{n \rightarrow \infty} \frac{f\left(2^{n} x\right)}{16^{n}}
$$

exists for all $x \in X$ and defines a unique quartic mapping $Q: X \rightarrow Y$ such that

$$
\|f(x)-Q(x)\| \leq \frac{1}{|16|-|16| L} \max \left\{\frac{1}{|22|} \gamma(0, x),\left|\frac{6}{11}\right| \gamma(x, x)\right\} .
$$

Proof. Putting $x=0$ in (2.5), we have

$$
\|12 f(3 \gamma)-70 f(2 \gamma)+148 f(\gamma)\| \leq \gamma(0, \gamma)
$$

for all $y \in X$.

Substituting $x=y$ in (2.5), we get

$$
\|f(3 \gamma)-4 f(2 \gamma)-17 f(\gamma)\| \leq \gamma(\gamma, \gamma)
$$

for all $y \in X$. By (3.3) and (3.4), we have

$$
\begin{aligned}
\|f(2 \gamma)-16 f(y)\| & =\left\|\frac{-1}{22}[12 f(3 \gamma)-70 f(2 \gamma)+148 f(y)]+\frac{6}{11}[f(3 \gamma)-4 f(2 \gamma)-17 f(y)]\right\| \\
& \leq \max \left\{\frac{1}{|22|} \gamma(0, y),\left|\frac{6}{11}\right| \gamma(y, y)\right\}
\end{aligned}
$$

for all $y \in X$. Consider the set

$$
S:=\{g: X \rightarrow Y, g(0)=0\}
$$

and the generalized metric $d$ in $S$ defined by

$$
d(f, g)=\inf _{\mu \in(0,+\infty)}\left\{\|g(x)-h(x)\| \leq \mu \max \left\{\frac{1}{|22|} \gamma(0, x),\left|\frac{6}{11}\right| \gamma(x, x)\right\}, \forall x \in X\right\}
$$

where inf $\varnothing=+\infty$. It is easy to show that $(S, d)$ is complete (see [[42], Lemma 2.1]). 
Now, we consider a linear mapping $J: S \rightarrow S$ such that

$$
J g(x):=\frac{1}{16} g(2 x)
$$

for all $x \in X$. It follows from (3.5) that

$$
d(f, J f) \leq \frac{1}{|16|}
$$

By Theorem 1.1, there exists a mapping $Q: X \rightarrow Y$ satisfying the following:

(1) $Q$ is a fixed point of $J$, that is,

$$
16 Q(x)=Q(2 x)
$$

for all $x \in X$. The mapping $Q$ is a unique fixed point of $J$ in the set

$$
\Omega=\{h \in S: d(g, h)<\infty\} .
$$

This implies that $Q$ is a unique mapping satisfying (3.8) such that there exists $\mu \in(0$, $\infty)$ satisfying

$$
\|f(x)-Q(x)\| \leq \mu \max \left\{\frac{1}{|22|} \gamma(0, x),\left|\frac{6}{11}\right| \gamma(x, x)\right\}
$$

for all $x \in X$.

(2) $d\left(J^{n} f, Q\right) \rightarrow 0$ as $n \rightarrow \infty$. This implies the equality

$$
\lim _{n \rightarrow \infty} \frac{f\left(2^{n} x\right)}{16^{n}}=Q(x)
$$

for all $x \in X$.

(3) $d(f, Q) \leq \frac{d(f, I f)}{1-L}$ with $f \in \Omega$, which implies the inequality

$$
d(f, C) \leq \frac{1}{|16|-|16| L}
$$

This implies that the inequality (3.2) holds. The rest of the proof is similar to the proof of Theorem 2.1.

Corollary 3.1. Let $\theta \geq 0$ and $r$ be a real number with $r>1$. Let $f: X \rightarrow Y$ be an even mapping with $f(0)=0$ satisfying (2.15). Then, the limit $Q(x)=\lim _{n \rightarrow \infty} \frac{f\left(2^{n} x\right)}{16^{n}}$ exists for all $x \in X$ and $Q: X \rightarrow Y$ is a unique quartic mapping such that

$$
\|f(x)-Q(x)\| \leq \frac{1}{|16|-|16|^{r+1}} \max \left\{\frac{\theta\|x\|^{r}}{|22|}, 2\left|\frac{6}{11}\right| \theta\|x\|^{r}\right\}
$$

for all $x \in X$.

Proof. The proof follows from Theorem 3.1 if we take

$$
\gamma(x, y)=\theta\left(\|x\|^{r}+\|y\|^{r}\right)
$$

for all $x, y \in X$. In fact, if we choose $L=|16|^{r}$, then we get the desired result. $\quad \square$ Similarly, we can obtain the following. We will omit the proof.

Theorem 3.2. Let $X$ be a non-Archimedean normed space and $Y$ a non-Archimedean Banach space. Assume that $\gamma: X^{2} \rightarrow[0, \infty)$ is a function such that there exists an $L<1$ with 


$$
\gamma\left(\frac{x}{2}, \frac{y}{2}\right) \leq \frac{L}{|16|} \gamma(x, y)
$$

for all $x, y \in X$. If $f: X \rightarrow Y$ is an even mapping with $f(0)=0$ satisfying (2.5), then the limit

$$
Q(x):=\lim _{n \rightarrow \infty} 16^{n} f\left(\frac{x}{2^{n}}\right)
$$

exists for all $x \in X$ and defines a unique quartic mapping $Q: X \rightarrow Y$ such that

$$
\|f(x)-Q(x)\| \leq \frac{L}{|16|-|16| L} \max \left\{\frac{1}{|22|} \gamma(0, x),\left|\frac{6}{11}\right| \gamma(x, x)\right\} .
$$

Corollary 3.2. Let $\theta \geq 0$ and $r$ be a real number with $0<r<1$. Let $f: X \rightarrow Y$ be an even mapping with $f(0)=0$ satisfying (2.15). Then, the limit $Q(x)=\lim _{n \rightarrow \infty} 16^{n} f\left(\frac{x}{2^{n}}\right)$ exists for all $x \in X$ and $Q: X \rightarrow Y$ is a unique quartic mapping such that

$$
\|f(x)-Q(x)\| \leq \frac{|16|}{|16|^{r+1}-|16|^{2}} \max \left\{\frac{\theta\|x\|^{r}}{|22|}, 2\left|\frac{6}{11}\right| \theta\|x\|^{r}\right\}
$$

for all $x \in X$.

Proof. The proof follows from Theorem 3.2 if we take

$$
\gamma(x, y)=\theta\left(\|x\|^{r}+\|y\|^{r}\right)
$$

for all $x, y \in X$. In fact, if we choose $L=|16|^{1-r}$, then we get the desired result.

\section{Non-Archimedean stability of Equation (1.1): a direct method-odd case}

Throughout this section, using direct method, we prove the generalized Hyers-Ulam stability of the functional Equation (1.1) in non-Archimedean spaces for an odd case.

Theorem 4.1. Let $G$ be an additive semigroup and $X$ a complete non-Archimedean space. Assume that $\phi: G^{2} \rightarrow[0,+\infty)$ is a function such that

$$
\lim _{n \rightarrow \infty}|8|^{n} \varphi\left(\frac{x}{2^{n}}, \frac{y}{2^{n}}\right)=0
$$

for all $x, y \in G$. Let for all $x \in G$

$$
\Phi(x)=\lim _{n \rightarrow \infty} \max \left\{|8|^{k+1} \max \left\{\frac{1}{|11|} \varphi\left(\frac{x}{2^{k}}, \frac{x}{2^{k+1}}\right),\left|\frac{14}{33}\right| \varphi\left(\frac{x}{2^{k+1}}, 0\right)\right\} ; 0 \leq k<n\right\}
$$

exist. Suppose that $f: G \rightarrow X$ is an odd mapping satisfying the inequality

$$
\left\|\Phi_{f}(x, y)\right\|_{X} \leq \varphi(x, y)
$$

for all $x, y \in G$. Then the limit

$$
C(x):=\lim _{n \rightarrow \infty} 8^{n}\left(f\left(\frac{x}{2^{n-1}}\right)-2 f\left(\frac{x}{2^{n}}\right)\right)
$$

exists for all $x \in G$ and $C: G \rightarrow X$ is a cubic mapping satisfying

$$
\|f(2 x)-2 f(x)-C(x)\|_{X} \leq \frac{1}{|8|} \Phi(x)
$$


for all $x \in$ G. Moreover, if

$$
\lim _{j \rightarrow \infty} \lim _{n \rightarrow \infty} \max \left\{|8|^{k+1} \max \left\{\frac{1}{|11|} \varphi\left(\frac{x}{2^{k^{\prime}}}, \frac{x}{2^{k+1}}\right),\left|\frac{14}{33}\right| \varphi\left(\frac{x}{2^{k+1}}, 0\right)\right\} ; j \leq k<n+j\right\}=0,
$$

then $C$ is the unique mapping satisfying (4.4).

Proof. Proceeding as in the proof of Theorem 2.1, we obtain

$$
\|f(4 \gamma)-10 f(2 \gamma)+16 f(\gamma)\|_{X} \leq \max \left\{\frac{1}{|11|} \varphi(2 \gamma, y),\left|\frac{14}{33}\right| \varphi(\gamma, 0)\right\}
$$

for all $y \in X$. Letting $y:=\frac{x}{2}$ and $g(x):=f(2 x)-2 f(x)$ for all $x \in X$, we get

$$
\left\|g(x)-8 g\left(\frac{x}{2}\right)\right\|_{X} \leq \max \left\{\frac{1}{|11|} \varphi\left(x, \frac{x}{2}\right),\left|\frac{14}{33}\right| \varphi\left(\frac{x}{2}, 0\right)\right\} .
$$

Replacing $x$ by $\frac{x}{2^{n}}$ in (4.6), we get

$$
\left\|8^{n} g\left(\frac{x}{2^{n}}\right)-8^{n+1} g\left(\frac{x}{2^{n+1}}\right)\right\|_{X} \leq|8|^{n} \max \left\{\frac{1}{|11|} \varphi\left(\frac{x}{2^{n}}, \frac{x}{2^{n+1}}\right),\left|\frac{14}{33}\right| \varphi\left(\frac{x}{2^{n+1}}, 0\right)\right\} .
$$

It follows from (4.1) and (4.7) that the sequence $\left\{8^{n} g\left(\frac{x}{2^{n}}\right)\right\}_{n=1}^{\infty}$ is a Cauchy sequence. Since $X$ is complete, so $\left\{8^{n} g\left(\frac{x}{2^{n}}\right)\right\}_{n=1}^{\infty}$ is convergent. Set

$$
C(x):=\lim _{n \rightarrow \infty} 8^{n} g\left(\frac{x}{2^{n}}\right)=\lim _{n \rightarrow \infty} 8^{n}\left(f\left(\frac{x}{2^{n-1}}\right)-2 f\left(\frac{x}{2^{n}}\right)\right) .
$$

Using induction, we see that

$$
\begin{aligned}
& \left\|8^{n} g\left(\frac{x}{2^{n}}\right)-g(x)\right\|_{X} \\
& \leq \frac{1}{|8|} \max \left\{|8|^{k+1} \max \left\{\frac{1}{|11|} \varphi\left(\frac{x}{2^{k}}, \frac{x}{2^{k+1}}\right),\left|\frac{14}{33}\right| \varphi\left(\frac{x}{2^{k+1}}, 0\right)\right\} ; 0 \leq k<n\right\} .
\end{aligned}
$$

By taking $n$ to approach infinity in (4.8), one obtains (4.4). If $L$ is another mapping satisfying (4.4), then, for $x \in G$, we get

$$
\begin{aligned}
& \|C(x)-L(x)\|_{X} \\
& =\lim _{j \rightarrow \infty}\left\|8^{j} L\left(\frac{x}{2^{j}}\right)-8^{j} C\left(\frac{x}{2^{j}}\right)\right\|_{X} \\
& =\lim _{j \rightarrow \infty}\left\|8^{j} L\left(\frac{x}{2^{j}}\right) \pm 8^{j} g\left(\frac{x}{2^{j}}\right)-8^{j} C\left(\frac{x}{2^{j}}\right)\right\|_{X} \\
& \leq \lim _{j \rightarrow \infty} \max \left\{\left\|8^{j}\left[L\left(\frac{x}{2^{j}}\right)-g\left(\frac{x}{2^{j}}\right)\right]\right\|_{X^{\prime}}\left\|8^{j}\left[g\left(\frac{x}{2^{j}}\right)-C\left(\frac{x}{2^{j}}\right)\right]\right\| \|_{X}\right\} \\
& \leq \frac{1}{|8|} \lim _{j \rightarrow \infty} \lim _{n \rightarrow \infty} \max \left\{|8|^{k+1} \max \left\{\frac{1}{|11|} \varphi\left(\frac{x}{2^{k^{\prime}}} \frac{x}{2^{k+1}}\right),\left|\frac{14}{33}\right| \varphi\left(\frac{x}{2^{k+1}}, 0\right)\right\} ; j \leq k<n+j\right\} \\
= & 0 .
\end{aligned}
$$

Therefore, $L=C$. This completes the proof. $\quad \square$

Corollary 4.1. Let $\xi:[0, \infty) \rightarrow[0, \infty)$ be a function satisfying

$$
\xi\left(\frac{t}{|2|}\right) \leq \xi\left(\frac{1}{|2|}\right) \xi(t), \quad \xi\left(\frac{1}{|2|}\right)<\frac{1}{|8|}
$$


for all $t \geq 0$. Let $\delta>0$ and $f: G \rightarrow X$ be an odd mapping satisfying the inequality

$$
\left\|\Phi_{f}(x, y)\right\|_{X} \leq \delta(\xi(|x|)+\xi(|y|))
$$

for all $x, y \in G$. Then the limit $C(x)=\lim _{n \rightarrow \infty} 8^{n}\left(f\left(\frac{x}{2^{n-1}}\right)-2 f\left(\frac{x}{2^{n}}\right)\right)$ exists for all $x \in$ $G$ and $C: G \rightarrow X$ is a unique cubic mapping such that

$$
\|f(2 x)-2 f(x)-C(x)\|_{X} \leq \max \left\{\frac{1}{|11|} \delta \xi(|x|)\left(1+\frac{1}{|8|}\right),\left|\frac{7}{132}\right| \xi(|x|)\right\}
$$

for all $x \in G$.

Proof. Defining $\phi: G^{2} \rightarrow[0, \infty)$ by $\phi(x, y):=\delta(\xi(|x|)+\xi(|y|))$. Since $|8| \xi\left(\frac{1}{|2|}\right)<1$, we have

$$
\lim _{n \rightarrow \infty}|8|^{n} \varphi\left(\frac{x}{2^{n}}, \frac{y}{2^{n}}\right) \leq \lim _{n \rightarrow \infty}\left[|8| \xi\left(\frac{1}{|2|}\right)\right]^{n} \varphi(x, y)=0
$$

for all $x, y \in G$. Also for all $x \in G$

$$
\begin{aligned}
\Phi(x) & =\lim _{n \rightarrow \infty} \max \left\{|8|^{k+1} \max \left\{\frac{1}{|11|} \varphi\left(\frac{x}{2^{k}}, \frac{x}{2^{k+1}}\right),\left|\frac{14}{33}\right| \varphi\left(\frac{x}{2^{k+1}}, 0\right)\right\} ; 0 \leq k<n\right\} \\
& =|8| \max \left\{\frac{1}{|11|} \varphi\left(x, \frac{x}{2}\right),\left|\frac{14}{33}\right| \varphi\left(\frac{x}{2}, 0\right)\right\} \\
& =|8| \max \left\{\frac{1}{|11|} \delta \xi(|x|)\left(1+\frac{1}{|8|}\right),\left|\frac{7}{132}\right| \xi(|x|)\right\}
\end{aligned}
$$

exists for all $x \in G$. On the other hand,

$$
\begin{aligned}
& \lim _{j \rightarrow \infty} \lim _{n \rightarrow \infty} \max \left\{|8|^{k+1} \max \left\{\frac{1}{|11|} \varphi\left(\frac{x}{2^{k}}, \frac{x}{2^{k+1}}\right),\left|\frac{14}{33}\right| \varphi\left(\frac{x}{2^{k+1}}, 0\right)\right\} ; j \leq k<n+j\right\} \\
& \lim _{j \rightarrow \infty}|8|^{j+1} \max \left\{\frac{1}{|11|} \varphi\left(\frac{x}{2^{j}}, \frac{x}{2^{j+1}}\right),\left|\frac{14}{33}\right| \varphi\left(\frac{x}{2^{j+1}}, 0\right)\right\} \\
& =0 .
\end{aligned}
$$

Applying Theorem 4.1, we get the desired result. $\square$

Theorem 4.2. Let $G$ be an additive semigroup and $X$ a complete non-Archimedean space. Assume that $\phi: G^{2} \rightarrow[0,+\infty)$ is a function such that

$$
\lim _{n \rightarrow \infty} \frac{\varphi\left(2^{n} x, 2^{n} y\right)}{|8|^{n}}=0
$$

for all $x, y \in G$. Let for each $x \in G$

$$
\Phi(x)=\lim _{n \rightarrow \infty} \max \left\{\frac{1}{|8|^{k+1}} \max \left\{\frac{1}{|11|} \varphi\left(2^{k+1} x, 2^{k} x\right),\left|\frac{14}{33}\right| \varphi\left(2^{k} x, 0\right)\right\} ; 0 \leq k<n\right\}
$$

exist. Suppose that $f: G \rightarrow X$ is an odd mapping satisfying the inequality (4.3). Then the limit

$$
C(x):=\lim _{n \rightarrow \infty} \frac{f\left(2^{n+1} x\right)-2 f\left(2^{n} x\right)}{8^{n}}
$$


exists for all $x \in G$ and $C: G \rightarrow X$ is a cubic mapping satisfying

$$
\|f(2 x)-2 f(x)-C(x)\|_{X} \leq \Phi(x)
$$

for all $x \in$ G. Moreover, if

$$
\lim _{j \rightarrow \infty} \lim _{n \rightarrow \infty} \max \left\{\frac{1}{|8|^{k+1}} \max \left\{\frac{1}{|11|} \varphi\left(2^{k+1} x, 2^{k} x\right),\left|\frac{14}{33}\right| \varphi\left(2^{k} x, 0\right)\right\} ; j \leq k<n+j\right\}=0,
$$

then $C$ is the unique mapping satisfying (4.12).

Proof. It follows from (4.5) that

$$
\left\|\frac{g(2 x)}{8}-g(x)\right\|_{X} \leq \frac{1}{|8|} \max \left\{\frac{1}{|11|} \varphi(2 x, x),\left|\frac{14}{33}\right| \varphi(x, 0)\right\}
$$

for all $x \in$ G. Replacing $x$ by $2^{n} x$ in (4.13), we get

$$
\left\|\frac{g\left(2^{n+1} x\right)}{8^{n+1}}-\frac{g\left(2^{n} x\right)}{8^{n}}\right\|_{X} \leq \frac{1}{|8|^{n+1}} \max \left\{\frac{1}{|11|} \varphi\left(2^{n+1} x, 2^{n} x\right),\left|\frac{14}{33}\right| \varphi\left(2^{n} x, 0\right)\right\} .
$$

It follows from (4.10) and (4.14) that the sequence $\left\{\frac{g\left(2^{n} x\right)}{8^{n}}\right\}_{n=1}^{\infty}$ is a Cauchy sequence. Since $X$ is complete, $\left\{\frac{g\left(2^{n} x\right)}{8^{n}}\right\}_{n=1}^{\infty}$ is convergent. It follows from (4.14) that

$$
\begin{aligned}
\left\|\frac{g\left(2^{p} x\right)}{8^{p}}-\frac{g\left(2^{q} x\right)}{8^{q}}\right\|_{X} & =\left\|\sum_{k=p}^{q-1} \frac{g\left(2^{k+1} x\right)}{8^{k+1}}-\frac{g\left(2^{k} x\right)}{8^{k}}\right\|_{X} \\
& \leq \max \left\{\left\|\frac{g\left(2^{k+1} x\right)}{8^{k+1}}-\frac{g\left(2^{k} x\right)}{8^{k}}\right\|_{X} ; p \leq k<q-1\right\} \\
& \leq \max \left\{\frac{1}{\mid 8^{k+1}} \max \left\{\frac{1}{|11|} \varphi\left(2^{k+1} x, 2^{k} x\right),\left|\frac{14}{33}\right| \varphi\left(2^{k} x, 0\right)\right\} ; p \leq k<q-1\right\}
\end{aligned}
$$

for all $x \in G$ and all non-negative integers $q, p$ with $q>p \geq 0$. Letting $p=0$ and passing the limit $q \rightarrow \infty$ in the last inequality, we obtain (4.12).

The rest of the proof is similar to the proof of Theorem 4.1.

Corollary 4.2. Let $\xi:[0, \infty) \rightarrow[0, \infty)$ be a function satisfying

$$
\xi(|2| t) \leq \xi(|2|) \xi(t), \quad \xi(|2|)<|8|
$$

for all $t \geq 0$. Let $\delta>0$ and $f: G \rightarrow X$ be a mapping satisfying the inequality (4.9). Then the limit $C(x)=\lim _{n \rightarrow \infty} \frac{f\left(2^{n+1} x\right)-2 f\left(2^{n} x\right)}{8^{n}}$ exists for all $x \in G$ and $C: G \rightarrow X$ is a unique cubic mapping such that

$$
\|f(2 x)-2 f(x)-C(x)\|_{X} \leq \frac{1}{|8|} \max \left\{\frac{1+|8|}{|11|} \delta \xi(|x|),\left|\frac{14}{33}\right| \delta \xi(|x|)\right\}
$$

for all $x \in G$.

Proof. Define $\phi: G^{2} \rightarrow[0, \infty)$ by $\phi(x, y):=\delta(\xi(|x|)+\xi(|y|))$. Proceeding as in the proof of Corollary 4.1, we have

$$
\lim _{n \rightarrow \infty} \frac{\varphi\left(2^{n} x, 2^{n} y\right)}{|8|^{n}}=0
$$


for all $x, y \in G$. Also

$$
\begin{aligned}
\Phi(x) & =\lim _{n \rightarrow \infty} \max \left\{\frac{1}{|8|^{k+1}} \max \left\{\frac{1}{|11|} \varphi\left(2^{k+1} x, 2^{k} x\right),\left|\frac{14}{33}\right| \varphi\left(2^{k} x, 0\right)\right\} ; 0 \leq k<n\right\} \\
& =\frac{1}{|8|} \max \left\{\frac{1}{|11|} \varphi(2 x, x),\left|\frac{14}{33}\right| \varphi(x, 0)\right\} \\
& \leq \frac{1}{|8|} \max \left\{\frac{1+|8|}{|11|} \delta \xi(|x|),\left|\frac{14}{33}\right| \delta \xi(|x|)\right\}
\end{aligned}
$$

exists for all $x \in G$. Applying Theorem 4.2, we get the desired result. $\quad \square$

Theorem 4.3. Let $G$ be an additive semigroup and $X$ a complete non-Archimedean space. Assume that $\phi: G^{2} \rightarrow[0,+\infty)$ is a function such that

$$
\lim _{n \rightarrow \infty}|2|^{n} \varphi\left(\frac{x}{2^{n}}, \frac{y}{2^{n}}\right)=0
$$

for all $x, y \in G$. Let for all $x \in G$

$$
\Phi(x)=\lim _{n \rightarrow \infty} \max \left\{|2|^{k} \max \left\{\frac{1}{|11|} \varphi\left(\frac{x}{2^{k^{k}}}, \frac{x}{2^{k+1}}\right),\left|\frac{14}{33}\right| \varphi\left(\frac{x}{2^{k+1}}, 0\right)\right\} ; 0 \leq k<n\right\}
$$

exist. Suppose that $f: G \rightarrow X$ is an odd mapping satisfying the inequality (4.3). Then the limit

$$
A(x):=\lim _{n \rightarrow \infty} 2^{n}\left(f\left(\frac{x}{2^{n-1}}\right)-8 f\left(\frac{x}{2^{n}}\right)\right)
$$

exists for all $x \in G$ and $A: G \rightarrow X$ is an additive mapping satisfying

$$
\|f(2 x)-8 f(x)-A(x)\|_{X} \leq \Phi(x)
$$

for all $x \in$ G. Moreover, if

$$
\lim _{j \rightarrow \infty} \lim _{n \rightarrow \infty} \max \left\{|2|^{k} \max \left\{\frac{1}{|11|} \varphi\left(\frac{x}{2^{k^{\prime}}}, \frac{x}{2^{k+1}}\right),\left|\frac{14}{33}\right| \varphi\left(\frac{x}{2^{k+1}}, 0\right)\right\} ; j \leq k<n+j\right\}=0,
$$

then $A$ is the unique mapping satisfying (4.19).

Proof. Letting $y:=\frac{x}{2}$ and $h(x):=f(2 x)-8 f(x)$ for all $x \in G$ in (4.5), we get

$$
\left\|h(x)-2 h\left(\frac{x}{2}\right)\right\|_{X} \leq \max \left\{\frac{1}{|11|} \varphi\left(x, \frac{x}{2}\right),\left|\frac{14}{33}\right| \varphi\left(\frac{x}{2}, 0\right)\right\} .
$$

Replacing $x$ by $\frac{x}{2^{n}}$ in (4.20), we obtain

$$
\begin{aligned}
& \left\|2^{n} h\left(\frac{x}{2^{n}}\right)-2^{n+1} h\left(\frac{x}{2^{n+1}}\right)\right\|_{X} \\
& \leq|2|^{n} \max \left\{\frac{1}{|11|} \varphi\left(\frac{x}{2^{n}}, \frac{x}{2^{n+1}}\right),\left|\frac{14}{33}\right| \varphi\left(\frac{x}{2^{n+1}}, 0\right)\right\} .
\end{aligned}
$$

Using induction, one can easily show that

$$
\begin{aligned}
& \left\|2^{n} h\left(\frac{x}{2^{n}}\right)-h(x)\right\|_{X} \\
& \quad \leq \max \left\{|2|^{k} \max \left\{\frac{1}{|11|} \varphi\left(\frac{x}{2^{k}}, \frac{x}{2^{k+1}}\right),\left|\frac{14}{33}\right| \varphi\left(\frac{x}{2^{k+1}}, 0\right)\right\} ; 0 \leq k<n\right\} .
\end{aligned}
$$

The rest of the proof is similar to the proof of Theorem 4.1. 
Corollary 4.3. Let $\xi:[0, \infty) \rightarrow[0, \infty)$ be a function satisfying

$$
\xi\left(\frac{t}{|2|}\right) \leq \xi\left(\frac{1}{|2|}\right) \xi(t), \quad \xi\left(\frac{1}{|2|}\right)<\frac{1}{|2|}
$$

for all $t \geq 0$. Let $\delta>0$ and $f: G \rightarrow X$ be an odd mapping satisfying the inequality (4.9). Then the limit $A(x)=\lim _{n \rightarrow \infty} 2^{n}\left(f\left(\frac{x}{2^{n-1}}\right)-8 f\left(\frac{x}{2^{n}}\right)\right)$ exists for all $x \in G$ and $A$ : $G \rightarrow X$ is a unique additive mapping such that

$$
\|f(2 x)-8 f(x)-A(x)\|_{X} \leq \max \left\{\left(1+\frac{1}{|2|}\right) \frac{\delta \xi(|x|)}{|11|},\left|\frac{7}{33}\right| \xi(|x|)\right\}
$$

for all $x \in G$.

Proof. Define $\phi: G^{2} \rightarrow[0, \infty)$ by $\phi(x, y):=\delta((\xi(|x|)+\xi(|y|))$. Also

$$
\begin{aligned}
\Phi(x) & =\lim _{n \rightarrow \infty} \max \left\{|2|^{k} \max \left\{\frac{1}{|11|} \varphi\left(\frac{x}{2^{k^{\prime}}}, \frac{x}{2^{k+1}}\right),\left|\frac{14}{33}\right| \varphi\left(\frac{x}{2^{k+1}}, 0\right)\right\} ; 0 \leq k<n\right\} \\
& =\max \left\{\left(1+\frac{1}{|2|}\right) \frac{\delta \xi(|x|)}{|11|},\left|\frac{7}{33}\right| \xi(|x|)\right\}
\end{aligned}
$$

exists for all $x \in G$. Applying Theorem 4.3, we get the desired result.

Similarly, we can obtain the following. We will omit the proof.

Theorem 4.4. Let $G$ be an additive semigroup and $X$ a complete non-Archimedean space. Assume that $\phi: G^{2} \rightarrow[0,+\infty)$ is a function such that

$$
\lim _{n \rightarrow \infty} \frac{\varphi\left(2^{n} x, 2^{n} y\right)}{|2|^{n}}=0
$$

for all $x, y \in G$. Let for each $x \in G$

$$
\Phi(x)=\lim _{n \rightarrow \infty} \max \left\{\frac{1}{|2|^{k}} \max \left\{\frac{1}{|11|} \varphi\left(2^{k+1} x, 2^{k} x\right),\left|\frac{14}{33}\right| \varphi\left(2^{k} x, 0\right)\right\} ; 0 \leq k<n\right\} 4
$$

exist. Suppose that $f: G \rightarrow X$ be an odd mapping satisfying the inequality (4.3). Then the limit

$$
A(x):=\lim _{n \rightarrow \infty} \frac{f\left(2^{n+1} x\right)-8 f\left(2^{n} x\right)}{2^{n}}
$$

exists for all $x \in G$ and $A: G \rightarrow X$ is an additive mapping satisfying

$$
\|f(2 x)-8 f(x)-A(x)\|_{X} \leq \frac{1}{|2|} \Phi(x)
$$

for all $x \in$ G. Moreover, if

$$
\lim _{j \rightarrow \infty} \lim _{n \rightarrow \infty} \max \left\{\frac{1}{|2|^{k}} \max \left\{\frac{1}{|11|} \varphi\left(2^{k+1} x, 2^{k} x\right),\left|\frac{14}{33}\right| \varphi\left(2^{k} x, 0\right)\right\} ; j \leq k<n+j\right\}=0,
$$

then $A$ is the unique mapping satisfying (4.25).

\section{Non-Archimedean stability of Equation (1.1): a direct method-even case}

Theorem 5.1. Let $G$ be an additive semigroup and $X$ a complete non-Archimedean space. Assume that $\phi: G^{2} \rightarrow[0,+\infty)$ is a function such that 


$$
\lim _{n \rightarrow \infty} \frac{\varphi\left(2^{n} x, 2^{n} y\right)}{|16|^{n}}=0
$$

for all $x, y \in G$. Let for all $x \in G$

$$
\Phi(x)=\lim _{n \rightarrow \infty} \max \left\{\frac{1}{|16|^{k}} \max \left\{\frac{1}{|22|} \varphi\left(0,2^{k} x\right),\left|\frac{6}{11}\right| \varphi\left(2^{k} x, 2^{k} x\right)\right\} ; 0 \leq k<n\right\}
$$

exist. Suppose that $f: G \rightarrow X$ is an even mapping with $f(0)=0$ satisfying the inequality (4.3). Then the limit

$$
Q(x):=\lim _{n \rightarrow \infty} \frac{f\left(2^{n} x\right)}{16^{n}}
$$

exists for all $x \in G$ and $Q: G \rightarrow X$ is a quartic mapping satisfying

$$
\|f(x)-Q(x)\|_{X} \leq \frac{1}{|16|} \Phi(x)
$$

for all $x \in$ G. Moreover, if

$$
\lim _{j \rightarrow \infty} \lim _{n \rightarrow \infty} \max \left\{\frac{1}{|16|^{k}} \max \left\{\frac{1}{|22|} \varphi\left(0,2^{k} x\right),\left|\frac{6}{11}\right| \varphi\left(2^{k} x, 2^{k} x\right)\right\} ; j \leq k<n+j\right\}=0,
$$

then $Q$ is the unique mapping satisfying (5.3).

Proof. Proceeding as in the proof of Theorem 3.1, we obtain

$$
\left\|\frac{f(2 x)}{16}-f(x)\right\|_{X} \leq \frac{1}{|16|} \max \left\{\frac{1}{|22|} \varphi(0, x),\left|\frac{6}{11}\right| \varphi(x, x)\right\} .
$$

One can easily show that

$$
\begin{aligned}
& \left\|\frac{f\left(2^{n} x\right)}{16^{n}}-f(x)\right\|_{X} \\
& \quad \leq \frac{1}{|16|} \max \left\{\frac{1}{|16|^{k}} \max \left\{\frac{1}{|22|} \varphi\left(0,2^{k} x\right),\left|\frac{6}{11}\right| \varphi\left(2^{k} x, 2^{k} x\right)\right\} ; 0 \leq k<n\right\} .
\end{aligned}
$$

The rest of the proof is similar to the proof of Theorem 4.1. $\quad$

Corollary 5.1. Let $\xi:[0, \infty) \rightarrow[0, \infty)$ be a function satisfying

$$
\xi(|2| t) \leq \xi(|2|) \xi(t), \quad \xi(|2|)<|16|
$$

for all $t \geq 0$. Let $\delta>0$ and $f: G \rightarrow X$ be an even mapping with $f(0)=0$ satisfying the inequality (4.9). Then the limit $Q(x)=\lim _{n \rightarrow \infty} \frac{f\left(2^{n} x\right)}{16^{n}}$ exists for all $x \in G$ and $Q: G \rightarrow X$ is a unique quartic mapping such that

$$
\|f(x)-Q(x)\|_{X} \leq \frac{1}{|16|} \max \left\{\frac{1}{|22|} \delta \xi(|x|), 2\left|\frac{6}{11}\right| \xi(|x|)\right\}
$$

for all $x \in G$.

Proof. Define $\phi: G^{2} \rightarrow[0, \infty)$ by $\phi(x, y):=\delta(\xi(|x|)+\xi(|y|))$. Also

$$
\Phi(x)=\max \left\{\frac{1}{|22|} \delta \xi(|x|), 2\left|\frac{6}{11}\right| \xi(|x|)\right\}
$$

exists for all $x \in G$. Applying Theorem 5.1, we get the desired result. $\quad \square$ 
Similarly, we can obtain the following. We will omit the proof.

Theorem 5.2. Let $G$ be an additive semigroup and $X$ a complete non-Archimedean space. Assume that $\phi: G^{2} \rightarrow[0,+\infty)$ is a function such that

$$
\left.\lim _{n \rightarrow \infty} 16\right|^{n} \varphi\left(\frac{x}{2^{n}}, \frac{y}{2^{n}}\right)=0
$$

for all $x, y \in G$. Let for all $x \in G$

$$
\Phi(x)=\lim _{n \rightarrow \infty} \max \left\{|16|^{k} \max \left\{\frac{1}{|22|} \varphi\left(0, \frac{x}{2^{k+1}}\right),\left|\frac{6}{11}\right| \varphi\left(\frac{x}{2^{k+1}}, \frac{x}{2^{k+1}}\right)\right\} ; 0 \leq k<n\right\}
$$

exist. Suppose that $f: G \rightarrow X$ is an even mapping satisfying the inequality (4.3). Then the limit

$$
Q(x):=\lim _{n \rightarrow \infty} 16^{n} f\left(\frac{x}{2^{n}}\right)
$$

exists for all $x \in G$ and $Q: G \rightarrow X$ is a quartic mapping satisfying

$$
\|f(x)-Q(x)\|_{X} \leq \Phi(x)
$$

for all $x \in G$. Moreover, if

$$
\lim _{j \rightarrow \infty} \lim _{n \rightarrow \infty} \max \left\{|16|^{k} \max \left\{\frac{1}{|22|} \varphi\left(0, \frac{x}{2^{k+1}}\right),\left|\frac{6}{11}\right| \varphi\left(\frac{x}{2^{k+1}}, \frac{x}{2^{k+1}}\right)\right\} ; j \leq k<n+j\right\}=0,
$$

then $Q$ is the unique mapping satisfying (5.4).

\section{Conclusion}

We linked here three different disciplines, namely, the non-Archimedean normed spaces, functional equations and fixed point theory. We established the generalized Hyers-Ulam stability of the functional Equation (1.1) in non-Archimedean normed spaces.

\section{Competing interests}

The authors declare that they have no competing interests.

\section{Authors' contributions}

All of the authors contributed equally and significantly in writing this paper. All authors read and approved the final manuscript.

\section{Acknowledgements}

The second and third authors were supported by Basic Science Research Program through the National Research Foundation of Korea funded by the Ministry of Education, Science and Technology (NRF-2010-0009232) and (NRF2009-0070788), respectively.

\section{Author details}

'Department of Mathematics, College of Sciences, Yasouj University, Yasouj 75914-353, Iran ${ }^{2}$ Department of Mathematics, Daejin University, Kyeonggi 487-711, Korea ${ }^{3}$ Department of Mathematics, Research Institute for Natural Sciences, Hanyang University, Seoul 133-791, Korea

Received: 6 June 2011 Accepted: 3 October 2011 Published: 3 October 2011

References

1. Ulam, SM: Problems in Modern Mathematics, Science Editions. Wiley, New York (1964)

2. Hyers, DH: On the stability of the linear functional equation. Proc Natl Acad Sci USA. 27, 222-224 (1941). doi:10.1073/ pnas.27.4.222 
3. Rassias, TM: On the stability of the linear mapping in Banach spaces. Proc Am Math Soc. 72, 297-300 (1978). doi:10.1090/S0002-9939-1978-0507327-1

4. Arriola, LM, Beyer, WA: Stability of the Cauchy functional equation over p-adic fields. Real Anal Exch. 31, 125-132 (2005)

5. Azadi Kenary, H: Non-Archimedean stability of Cauchy-Jensen type functional equation. Int J Nonlinear Anal Appl. 1(2), $1-10(2010)$

6. Azadi Kenary, H: Stability of a Pexiderial functional equation in random normed spaces. Rend Circ Mat Palermo

7. Azadi Kenary, H, Shafaat, Kh, Shafei, M, Takbiri, G: Hyers-Ulam-Rassias stability of the Appollonius quadratic mapping in RN-spaces. J Nonlinear Sci Appl. 4(1), 110-119 (2011)

8. Cho, Y, Kim, H: Stability of functional inequalities with Cauchy-Jensen additive mappings. Abstr Appl Anal 2007, 13 (2007). Article ID 89180

9. Găvruta, P: A generalization of the Hyers-Ulam-Rassias stability of approximately additive mappings. J Math Anal Appl. 184, 431-436 (1994). doi:10.1006/jmaa.1994.1211

10. Skof, F: Local properties and approximation of operators. Rend Sem Mat Fis Milano. 53, 113-129 (1983). doi:10.1007/ BF02924890

11. Cholewa, PW: Remarks on the stability of functional equations. Aequatines Math. 27, 76-86 (1984). doi:10.1007/ BF02192660

12. Czerwik, S: Functional Equations and Inequalities in Several Variables. World Scientific, River Edge, NJ (2002)

13. Gordji, ME, Savadkouhi, MB: Stability of mixed type cubic and quartic functional equations in random normed spaces. $J$ Inequal Appl 2009, 9 (2009). Article ID 527462

14. Gordji, ME, Savadkouhi, MB, Park, C: Quadratic-quartic functional equations in RN-spaces. J Inequal Appl 2009, 14 (2009). Article ID 868423

15. Gordji, ME, Khodaei, H: Stability of Functional Equations. Lap Lambert Academic Publishing, USA (2010)

16. Gordji, ME, Zolfaghari, S, Rassias, JM, Savadkouhi, MB: Solution and stability of a mixed type cubic and quartic functional equation in quasi-Banach spaces. Abstr Appl Anal 2009, 14 (2009). Article ID 417473

17. Fechner, W: Stability of a functional inequality associated with the Jordan-Von Neumann functional equation. Aequationes Math. 71, 149-161 (2006). doi:10.1007/s00010-005-2775-9

18. Hyers, DH, Isac, G, Rassias, TM: Stability of Functional Equations in Several Variables. Birkhäuser, Basel (1998)

19. Jung, S: Hyers-Ulam-Rassias Stability of Functional Equations in Mathematical Analysis. Hadronic Press, Palm Harbor (2001)

20. Khodaei, H, Rassias, TM: Approximately generalized additive functions in several variables. Int I Nonlinear Anal Appl. $1(1), 22-41$ (2010)

21. Kominek, Z: On a local stability of the Jensen functional equation. Demon Math. 22, 499-507 (1989)

22. Mirmostafaee, AK: Approximately additive mappings in non-Archimedean normed spaces. Bull Korean Math Soc. 46 , 387-400 (2009). doi:10.4134/BKMS.2009.46.2.387

23. Moradlou, F, Vaezi, H, Park, C: Fixed point and stability of an additive functional equation of $n$-Apollonius type in $C^{*}$ algebras. Abstr Appl Anal 2008, 13 (2008). Article ID 672618

24. Moslehian, MS, Rassias, TM: Stability of functional equations in non-Archimedean spaces. Appl Anal Discret Math. 1, 325-334 (2007). doi:10.2298/AADM0702325M

25. Park, C: Fuzzy stability of a functional equation associated with inner product spaces. Fuzzy Sets Syst. 160, 1632-1642 (2009). doi:10.1016/j.fss.2008.11.027

26. Park, C: Generalized Hyers-Ulam-Rassias stability of $n$-sesquilinear-quadratic mappings on Banach modules over $C^{*}$ algebras. J Comput Appl Math. 180, 279-291 (2005). doi:10.1016/j.cam.2004.11.001

27. Park, C: Fixed points and Hyers-Ulam-Rassias stability of Cauchy-Jensen functional equations in Banach algebras. Fixed Point Theory Appl 2007, 15 (2007). Article ID 50175

28. Park, C: Generalized Hyers-Ulam-Rassias stability of quadratic functional equations: a fixed point approach. Fixed Point Theory Appl 2008, 9 (2008). Art. ID 493751

29. Rassias, ThM: On the stability of functional equations and a problem of Ulam. Acta Appl Math. 62, 23-130 (2000). doi:10.1023/A:1006499223572

30. Saadati, R, Park, C: Non-Archimedean $\mathcal{L}$-fuzzy normed spaces and stability of functional equations. Comput Math Appl. 60, 2488-2496 (2010). doi:10.1016/j.camwa.2010.08.055

31. Saadati, $R$, Vaezpour, $M, C h o, Y: A$ note to paper on the stability of cubic mappings and quartic mappings in random normed spaces. J Inequal Appl 2009, 6 (2009). Article ID 214530

32. Saadati, R, Zohdi, MM, Vaezpour, SM: Nonlinear L-random stability of an ACQ functional equation. J Inequal Appl 2011, 23 (2011). Article ID 194394. doi:10.1186/1029-242X-2011-23

33. Hensel, K: Ubereine news begrundung der theorie der algebraischen Zahlen. Jahresber Deutsch Math Verein. 6, 83-88 (1897)

34. Deses, D: On the representation of non-Archimedean objects. Topol Appl. 153, 774-785 (2005). doi:10.1016/j. topol.2005.01.010

35. Katsaras, AK, Beoyiannis, A: Tensor products of non-Archimedean weighted spaces of continuous functions. Georgian Math J. 6, 33-44 (1999). doi:10.1023/A:1022926309318

36. Khrennikov, A: Non-Archimedean analysis: quantum paradoxes, dynamical systems and biological models. In Math Appl, vol. 427,Kluwer, Dordrecht (1997)

37. Nyikos, PJ: On some non-Archimedean spaces of Alexandrof and Urysohn. Topol Appl. 91, 1-23 (1999). doi:10.1016/ S0166-8641(97)00239-3

38. Diaz, J, Margolis, B: A fixed point theorem of the alternative for contractions on a generalized complete metric space. Bull Am Math Soc. 74, 305-309 (1968). doi:10.1090/50002-9904-1968-11933-0

39. Cădariu, L, Radu, V: Fixed points and the stability of Jensen's functional equation. J Inequal Pure Appl Math. 4, 1-9 (2003)

40. Lee, S, Im, S, Hwang, I: Quartic functional equations. J Math Anal Appl. 307, 387-394 (2005). doi:10.1016/j. jmaa.2004.12.062

41. Eshaghi-Gordji, M, Kaboli-Gharetapeh, S, Park, C, Zolfaghri, S: Stability of an additive-cubicquartic functional equation Adv Diff Equ 2009, 20 (2009). Article ID 395693 
42. Mihet, D, Radu, V: On the stability of the additive Cauchy functional equation in random normed spaces. J Math Anal Appl. 343, 567-572 (2008)

doi:10.1186/1687-1812-2011-60

Cite this article as: Azadi Kenary et al:: Nonlinear approximation of an ACQ-functional equation in nan-spaces.

Fixed Point Theory and Applications 2011 2011:60.

Submit your manuscript to a SpringerOpen ${ }^{\circ}$ journal and benefit from:

- Convenient online submission

- Rigorous peer review

- Immediate publication on acceptance

- Open access: articles freely available online

- High visibility within the field

- Retaining the copyright to your article

Submit your next manuscript at $>$ springeropen.com 